\title{
Lactococcus chungangensis CAU 28 alleviates diet-induced obesity and adipose tissue metabolism in vitro and in mice fed a high-fat diet
}

\author{
Qi Zhang, Jong-Hwa Kim, Yena Kim, and Wonyong Kim* (i) \\ Department of Microbiology, Chung-Ang University College of Medicine, Seoul 06974, Republic of Korea
}

\begin{abstract}
Obesity, which has become a major public health problem, can arise from complex dyslipidemia, insulin resistance, and immune responses, among other mechanisms. Some Lactobacillus strains effectively ameliorate obesity; however, the beneficial effects of Lactococcus spp., which are often used as dairy starters, remain unclear. In the present study, we evaluated the efficacy of Lactococcus chungangensis CAU 28 using the 3T3-L1 cell line and obese mice fed a high-fat diet. Overall, administration of Lc. chungangensis CAU 28 effectively resolved obesity associated with weight gain and lipid accumulation. In differentiated 3T3-L1 cells, Lc. chungangensis CAU 28 treatment significantly diminished the total lipid quantity, inhibited triglyceride formation, and prevented the proliferation of adipogenic transcription factors (fatty acid synthase, adiponectin, peroxisome proliferator-activated receptor-gamma, and CCAAT-enhancer-binding protein- $\alpha$ ) associated with lipid accumulation. In the obesity mouse model, wherein the intake of $L c$. chungangensis CAU 28 effectively reduced body weight gain, along with fat differentiation and accumulation (white fat; abdominal and subcutaneous). Furthermore, Lc. chungangensis CAU 28 increased serum adiponectin levels, decreased serum leptin levels, and effectively regulated adipokine secretion. It also increased the high-density lipoprotein:cholesterol ratio, reduced total cholesterol and triglyceride levels, reduced the low-density lipoprotein:cholesterol ratio, and affected obesity-regulated inflammatory cytokines IL-6, tumor necrosis factor- $\alpha$, IFN- $\gamma$, and IL-1 $\beta$. Additionally, Lc. chungangensis CAU 28 was associated with an increase in the $\mathrm{CD} 3^{+} \mathrm{CD} 4^{+} \mathrm{CD} 8^{-}$phenotype among obese mice. Thus, the administration of Lc. chungangensis CAU 28 induced antiobesity effects, suggesting potential applications of this species as a supplement for obesity mitigation.
\end{abstract}

Received April 8, 2020.

Accepted July 21, 2020.

*Corresponding author: kimwy@cau.ac.kr
Key words: Lactococcus chungangensis, obesity, adipokine, leptin, high-fat diet

\section{INTRODUCTION}

Obesity is a major public health problem that has recently become a worldwide pandemic (World Health Organization, 2000). In 2016, approximately 650 million adults (13\% of the world's adult population) and over 340 million children (aged 5-19 yr) were overweight or obese (World Health Organization, 2018). An association exists between obesity and increased risk of various chronic diseases, including hypertension, type 2 diabetes (Golubnitschaja and Costigliola, 2012), dyslipidemia, and cardiovascular diseases (Hursting et al., 2012; Jin et al., 2013). Many treatments exist for obesity control, including diet, exercise, behavior modifications, and medication (You et al., 2014). Among these, reducing excessive energy intake is one of the main approaches to addressing obesity; however, enforcing compliance with energy-restricted diets is difficult (Yu et al., 2009).

According to numerous studies, the increasing proportion of people with a body mass index (BMI) $\geq 25 \mathrm{~kg} /$ $\mathrm{m}^{2}$, blood pressure $>120 / 80 \mathrm{~mm} \mathrm{Hg}$, blood lipid levels $>20 \%$ of plasma, and abnormal lipoprotein levels [lowdensity lipoprotein (LDL) cholesterol $\geq 130 \mathrm{mg} / \mathrm{dL}$, and high-density lipoprotein (HDL) cholesterol $<40$ $\mathrm{mg} / \mathrm{dL}]$ indicates an increasing obesity trend worldwide (Rinaldi et al., 2012; Interator et al., 2017; Tran et al., 2017). Additionally, obesity elevates triglyceride (TG) and total cholesterol (TC) levels, increasing the incidence of heart disease (Golubnitschaja and Costigliola, 2012). In addition, fat accumulation in moderately obese individuals leads to secretion of proinflammatory cytokines, hyperinsulinemia, and insulin resistance. Obesity can lead to the formation of fatty liver lesions and induce fatty liver disease (Horowitz et al., 1999; Spor et al., 2011).

Probiotics are live microorganisms, which, when administered in adequate amounts, confer health benefits on the host (FAO/WHO, 2002). Probiotic lactic acid bacteria (such as those from the Lactobacillus genus and Bacillus natto) have been shown to work 
effectively against obesity and obesity-related metabolic syndromes, and their administration ameliorates the chronic low-grade inflammation associated with pathological aspects of obesity and metabolic syndrome (Miyoshi et al., 2014; Choi et al., 2020; Wang et al., 2020). Lactobacilli are the best-known probiotics and are critical constituents of the intestinal microflora (Kang et al., 2013). Moreover, only Lactococcus lactis exerts an antiobesity effect by regulating lipid metabolism and insulin resistance, reducing adipocyte size in white adipose tissue, and regulating adipokine secretion (Castaner et al., 2018; Nguyen et al., 2018; Naudin et al., 2020).

Lactococcus chungangensis CAU 28 was isolated from a wastewater treatment plant in Cheonan (Republic of Korea) as the sixth member of the Lactococcus genus (Cho et al., 2008). It has been found to exhibit enzymatic activities, including alcohol dehydrogenase, aldehyde dehydrogenase, amylase, proteinase, and lipase, which can be used to manufacture dairy products (Konkit et al., 2014, 2015, 2016; Konkit and Kim, 2016). Furthermore, Lc. chungangensis CAU 28 has been shown to inhibit the production of proinflammatory cytokines in atopic dermatitis-like skin lesions of Nc/Nga mice (Choi et al., 2016; Kim et al., 2019). However, the effects of oral administration of this strain in mice fed a high-fat diet have not yet been studied. In this study, we aimed to explore the effects of Lc. chungangensis CAU 28 on obesity-related variables, such as BW, fat differentiation and accumulation, and serum lipid, cytokine, and mRNA expression levels in a high-fat diet-induced obesity mouse model.

\section{MATERIALS AND METHODS}

\section{Preparation of Lysate of Lc. chungangensis CAU 28}

Lactococcus chungangensis CAU 28 was incubated at $30^{\circ} \mathrm{C}$ for $18 \mathrm{~h}$ on trypticase soy agar plates. After culturing, a single colony of Lc. chungangensis CAU 28 was inoculated in tryptic soy broth (BD BBL, Sparks, MD) and incubated. Then, Lc. chungangensis CAU 28 was harvested using centrifugation $(12,000 \times g$ for 20 min at $4^{\circ} \mathrm{C}$ ) and washed 3 times with PBS to eliminate any residual substances. To set the concentration to 1 $\times 10^{10}$ cells $/ \mathrm{mL}$, Lc. chungangensis $\mathrm{CAU} 28$ reconstituted with tryptic soy broth (BD BBL) was diluted 100 times with a matched optical density (OD) value $\left(1 \times 10^{8}\right.$ cells $\left./ \mathrm{mL}\right)$ using a microplate reader with the absorbance at $600 \mathrm{~nm}$. Then, to obtain bacteria cell extracts, sterilized beads were added and vortexed, followed by freezing and dissolving in liquid nitrogen 3 times. After centrifugation $(12,000 \times g$ for $20 \mathrm{~min}$ at $\left.4^{\circ} \mathrm{C}\right)$, the supernatants were aliquoted $(1 \mathrm{~mL}$ each $)$ in sterilized $1.5-\mathrm{mL}$ tubes and kept at $-80^{\circ} \mathrm{C}$ until use.

\section{Cell Culture and Stimulation of 3T3-L1 Adipocytes}

The standard method was used to culture 3T3-L1 preadipocytes, which were received from the American Type Culture Collection (Manassas, VA). Dulbecco's modified Eagle medium (DMEM) containing 1\% penicillin-streptomycin and 10\% bovine calf serum (Lonza, Walkersville, MD) was used to culture cells at $37^{\circ} \mathrm{C}$ in a $5 \% \mathrm{CO}_{2}$ incubator. 3T3-L1 cells were seeded at $3 \times 10^{3}$ cells/mL per well in a 12 -well plate and cultured until confluency.

For stimulation on the differentiation of 3T3-L1 adipocytes, cells were stimulated with methylisobutylxanthine-dexamethasone-insulin (MDI; 3-isobutyl1-methylxanthine, dexamethasone, and insulin) differentiation medium until d 3. An MDI cocktail medium consisting of $10 \%$ fetal bovine serum (FBS), $1 \%$ penicillin-streptomycin, $5 \mu \mathrm{g} / \mathrm{mL}$ insulin, $0.5 \mathrm{mM}$ 3-isobutyl-1-methylxanthine, and $1 \mu M$ dexamethasone (all from Sigma-Aldrich, St. Louis, MO). After differentiation on d 4, 3T3-L1 adipocytes were sustained in DMEM containing $10 \%$ FBS, $5 \mu \mathrm{g} / \mathrm{mL}$ insulin, and $1 \%$ penicillin-streptomycin until d 8 , and then cells were replaced with 10\% FBS in DMEM and 1\% penicillinstreptomycin until d 10. Additionally, medium was replaced every $2 \mathrm{~d}$. To elucidate the effects of lysates of Lc. chungangensis CAU 28 on the differentiation from preadipocyte to adipocyte, the lysates were treated at a concentration of $1 \times 10^{8}$ cells of lysates.

\section{Total Lipid Quantification by Oil Red O Staining}

To assess the lipid content of 3T3-L1 adipocyte, Oil red $\mathrm{O}$ staining was performed using the method outlined by Blessing et al. (2016), with Oil red O dissolved in isopropanol. Differentiated 3T3-L1 cells were washed 3 times using PBS and fixed for $1 \mathrm{~h}$ with $10 \%$ formaldehyde (Sigma-Aldrich) in PBS. After cells were fixed for $1 \mathrm{~h}$, washing was repeated, followed by staining with Oil red O (Sigma-Aldrich) solution for $1 \mathrm{~h}$ in a dark room at room temperature. Then, the cells were washed, the stained fat droplets were dissolved using isopropanol, and lipid was quantified by measuring the absorbance at $500 \mathrm{~nm}$. Stained fat droplets were microscopically photographed. Lipid accumulation was calculated by using the following formula: lipid accumulation $(\%)=$ $(\mathrm{A}-\mathrm{B} / \mathrm{C}) \times 100$, where $\mathrm{A}$ is the sample $\mathrm{OD}$ at 500 $\mathrm{nm}, \mathrm{B}$ is the blank OD at $500 \mathrm{~nm}$, and $\mathrm{C}$ is the control OD at $500 \mathrm{~nm}$ 


\section{Triglyceride Assay}

The TG content of the 3T3-L1 adipocytes was determined using a TG colorimetric assay kit (Cayman Chemical, Ann Arbor, MI) with the method described by Rudnicki et al. (2018). Differentiated 3T3-L1 cells were detached by $0.25 \%$ trypsin-EDTA. Detached cells were harvested in $1.5-\mathrm{mL}$ tubes and centrifuged at 1,000 $\times g$ for $10 \mathrm{~min}$ at $4^{\circ} \mathrm{C}$. After centrifugation, cell pellets were dissolved by diluted standard diluent supplied in TG colorimetric assay kit, and beads were added to 1.5-mL tubes for vortexing, followed by centrifugation $\left(10,000 \times g, 10 \mathrm{~min}, 4^{\circ} \mathrm{C}\right)$. The supernatant was separated into a new $1.5-\mathrm{mL}$ tube and stored at $-80^{\circ} \mathrm{C}$ until analysis.

\section{Quantification of Lipid Accumulation Factors by Real-Time PCR}

The mRNA expression levels of lipid accumulation factors were analyzed using total RNA extracted from cells. With methods described in previous publications (Fasshauer et al., 2003; Jeong, et al., 2010; Lee et al., 2011; Gwon et al., 2013), real-time PCR analysis was used to quantify fatty acid synthase $(F A S)$, adiponectin (ADIPOQ), peroxisome proliferator-activated receptorgamma $(P P A R G)$, and CCAAT-enhancer-binding protein- $\alpha(C E B P A)$, which are associated with lipid accumulation. Total RNA was extracted using TRI Reagent (Sigma-Aldrich), and $50 \mu \mathrm{L}$ of RNase-free water was added to a tube containing extracted total RNA that was kept at $-80^{\circ} \mathrm{C}$ until analysis. A NanoQuant Spectrophotometer (Infinite 200; Tecan, Männedorf, Switzerland) was used to check the extracted total RNA concentration, and $0.5 \mu \mathrm{g}$ of total RNA was reverse-transcribed into cDNA using a PrimeScript first-strand cDNA synthesis kit (Takara Bio, Shiga, Japan). To quantify the mRNA expression levels of lipid accumulation factors, real-time quantitative PCR was performed using a 7500 Fast Real-time PCR system and SYBR green PCR kit (Qiagen, Hilden, Germany). Fold changes were expressed using the $2^{-\Delta \Delta \mathrm{Ct}}$ method. Supplemental Table S1 (https://doi.org/10.3168/jds .2020-18681) lists 36B4 (control gene) and primers for lipid accumulation factors.

\section{Animal Model}

Six-week-old male C57BL/6J mice $(\mathrm{n}=40)$ fed a normal-fat diet (NFD, 10\% of calories obtained from fat) and a high-fat diet (HFD, $60 \%$ of calories obtained from fat) were purchased from the Central Lab Animal Incorporation (Seoul, Korea). All mice were housed in standard plastic cages in an animal room and main- tained under a 12-h light/dark cycle at a constant temperature of $22 \pm 1^{\circ} \mathrm{C}$ and at $50 \pm 10 \%$ relative humidity. Mice received filtered water and pelletized NFD for 1 wk after arrival; they were then randomly divided into 4 groups ( $\mathrm{n}=10$ in each group) as follows: (1) negative control group: fed NFD and orally administered PBS; (2) positive control group: fed HFD and orally administered PBS; (3) simvastatin group: fed HFD and orally administered simvastatin (a lipidlowering medication that also lowers leptin levels and can effect weight loss); and (4) CAU 28 group: fed HFD and orally administered freeze-dried Lc. chungangensis CAU 28.

Mice had free access to the diet and to sterile water throughout the experiment. Freeze-dried Lc. chungangensis CAU $28\left(1 \times 10^{8} \mathrm{cfu} /\right.$ mouse $)$ was suspended in $200 \mu \mathrm{L}$ of PBS and administered by oral gavage. The negative and positive control groups were administered equal volumes of PBS. Simvastatin (Sigma-Aldrich; 10 $\mathrm{mg} / \mathrm{kg}$ ) was dissolved in $200 \mu \mathrm{L}$ of PBS and administered orally (Zhao et al., 2019). All mice were orally gavaged once daily for $12 \mathrm{wk}$.

All protocols and procedures were approved by the Korean Food and Drug Administration (KFDA) guidelines. The animals used in the present study were cared for according to the principles and guidelines of the Chung-Ang University Institutional Animal Care and Use Committee (IACUC) of the Laboratory Animal Research Center (IACUC no. 2017-00044).

\section{BW, BMI, Body Fat, and Organ Weight}

Animal BW and body lengths (naso-anal length) were measured once a week. Each mouse's BW and body length were determined once per week, and BMI $\left[\mathrm{BW}(\mathrm{kg}) /\right.$ height $\left.^{2}\left(\mathrm{~m}^{2}\right)\right]$ was calculated at wk 12 . Immediately after the mice were killed, blood, organs (liver, lung, kidney, and spleen), and adipose tissue (abdominal, subcutaneous, and scapular fat) were harvested for further analysis. Organ weights were measured using an electronic weighing balance after calibrating the scale every time. The organs and tissues were rinsed with saline before further analysis.

\section{Hormone Analysis}

Blood samples were drawn by retro-orbital sinus puncture via the medial canthus of the eye. The blood samples were collected serum separate tubes (SST; Becton, Dickinson and Co., Franklin Lakes, NJ), left to coagulate for $1 \mathrm{~h}$ at $4^{\circ} \mathrm{C}$ and then centrifuged for 1 $\mathrm{h}$ at $5,000 \times \mathrm{g}$. The supernatant $($ serum) was stored at $-80^{\circ} \mathrm{C}$ until analysis. Concentrations of TC, HDL, LDL, and TG were then determined in the supernatant 
(blood serum), using the appropriate assay kits (Green Cross Biopharmaceutical, Yongin, Korea). The serum concentration of adiponectin was determined by ELISA, using a commercial adiponectin ELISA kit (Cusabio, Houston, TX), as described in the kit manual. Serum leptin concentrations were determined using a Linco Human Leptin ELISA kit (Linco Research, St. Charles, MO) following the manufacturer's instructions. Absorbance of samples was measured at $450 \mathrm{~nm}$ using an Infinite M200 NanoQuant plate reader (Tecan).

\section{Cytokine Analysis}

Blood serum samples were used for cytokine analyses: tumor necrosis factor (TNF)- $\alpha$, IFN- $\gamma$, IL-1 $1 \beta$, and IL-6. Cytokine concentrations were determined using an appropriate ELISA kit (R\&D Systems, Minneapolis, MN) according to the manufacturer's instructions. Sample absorbance at $450 \mathrm{~nm}$ was measured using an Infinite M200 NanoQuant plate reader (Tecan).

\section{Flow Cytometry Analysis}

The spleens were harvested from mice and crushed in a cell strainer (SPL Life Sciences, Pocheon, Korea). The cells were counted after staining with trypan blue using a TC10 automated cell counter (Bio-Rad, Hercules, CA). Then, they were diluted to $2.0 \times 10^{6}$ cells/tube and stained with phycoerythrin-labeled anti-mouse antibodies, CD3, CD4, or CD8 (BD Biosciences, San Jose, CA), on ice for $20 \mathrm{~min}$. The cells were analyzed by collecting at least 10,000 events in a FACSCalibur flow cytometer (BD Biosciences) and BD CellQuest Pro Software (version 6.0; BD Biosciences).

\section{Histological Analysis}

The hepatic, abdominal, subcutaneous, and scapular fat tissues were collected for histological examination. The adipose tissues were washed thoroughly with saline. The samples were portioned and fixed in $10 \%$ (vol/vol) neutral-buffered formalin for $24 \mathrm{~h}$ at room temperature. Then, they were embedded in paraffin and sectioned $(4-5 \mu \mathrm{m})$ for staining with hematoxylin and eosin. The size of the fat cells in adipose tissue was determined after examining 3 random fields of view (400× magnification) using light microscopy (Leica, Wetzlar, Germany).

\section{Statistical Analysis}

Results are presented as means \pm standard deviations. The data were normally distributed, and significant differences between groups were determined by one-way ANOVA with Tukey post hoc tests. A $P$-value $<0.05$ was considered statistically significant. All analyses were conducted using GraphPad Prism (version 7.0; GraphPad Software, La Jolla, CA).

\section{RESULTS}

\section{Effect of Lc. chungangensis CAU 28 on Adipocyte Differentiation and Intracellular TG Levels}

The effect of Lc. chungangensis CAU 28 on 3T3-L1 adipocyte differentiation was demonstrated by Oil red O staining (Figure 1A). In the cells treated with $L c$. chungangensis CAU 28, lipid accumulation occurred at $88.1 \%$ of the rate observed in the control cells. In other words, there was an 11.9-percentage- point decrease in lipid accumulation associated with Lc. chungangensis CAU 28 treatment compared with the cells treated only with MDI. Colorimetric analysis was performed to compare the intracellular TG contents and showed that Lc. chungangensis CAU 28 inhibited TG formation in 3T3-L1 cells (Figure 1B). The CAU 28 treatment was associated with a 69.9-percentage-point decrease in TG content compared with treatment with MDI only.

\section{Effect of Lc. chungangensis CAU 28 on Lipid Metabolism-Related Gene Expression}

The mRNA expression levels of different lipid metabolism-related factors (FAS, ADIPOQ, PPARG, and $C E B P A$ ) in differentiated 3T3-L1 adipocytes were dramatically reduced after Lc. chungangensis CAU 28 treatment relative to the levels observed in the cells treated with only MDI (Figure $1 \mathrm{C}-\mathrm{F}$ ). The mRNA expression levels of ADIPOQ and PPARG were $57.3 \%$ and $44.1 \%$ of the levels observed in the cells treated with only MDI, respectively. Moreover, expression of FAS and CEBPA were $75.2 \%$ and $65.3 \%$, respectively, in cells treated with Lc. chungangensis CAU 28 compared with that in cells treated with MDI alone.

\section{Effect of LC. chungangensis CAU 28 Intake on BMI, Organ Weight, and Relative Adipose:BW Ratios}

The initial BW of the mice in all groups did not differ significantly $(P=0.65)$. The area under the curve for BMI was significantly lower in the CAU 28 group than in the positive control $(P<0.05)$ and simvastatin $(P$ $<0.05$ ) groups (Figure 2A). However, there were no significant differences between the ratios for the liver, lung, or kidney to BW in the CAU 28 and simvastatin groups compared with the positive control group. The white fat:BW ratio was markedly reduced in the CAU 28 group compared with that in the positive control 
(A)

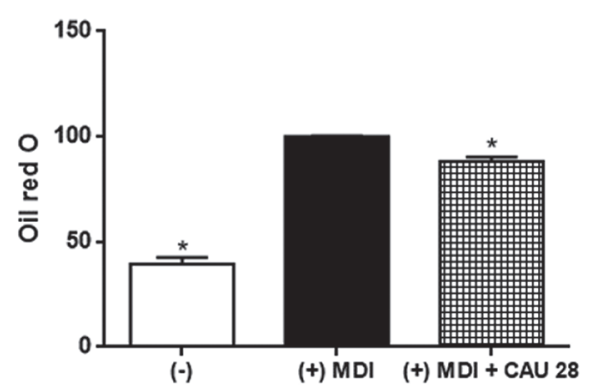

(C)

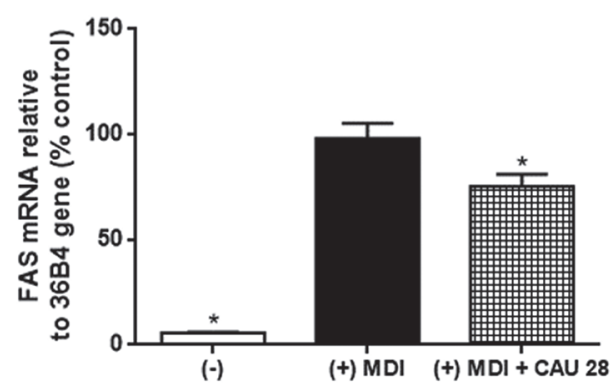

(E)

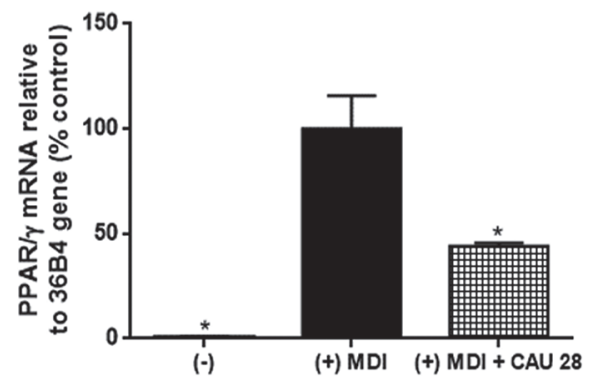

(B)

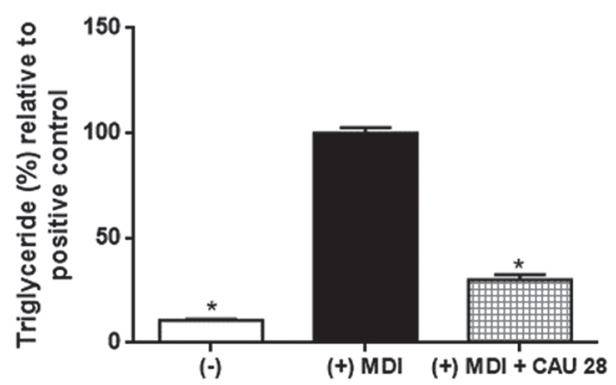

(D)

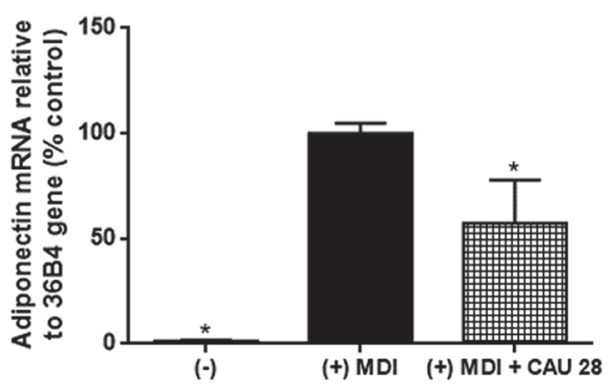

(F)

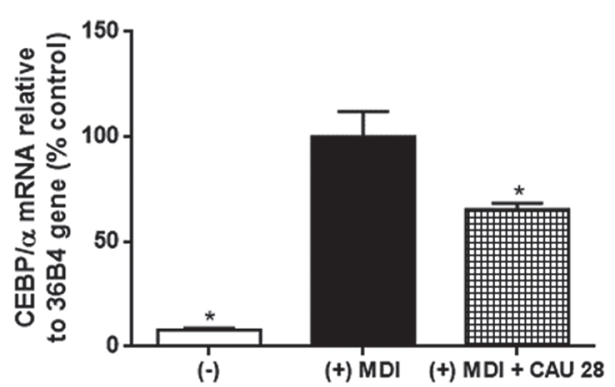

Figure 1. Effect of oral administration of Lactococcus chungangensis CAU 28 dry cells on adipocyte differentiation, intracellular triglyceride, and lipid metabolism-related factors. (A) Oil red $\mathrm{O}$ staining for adipocyte differentiation, (B) formation of triglycerides, and (C-F) lipid metabolism-related factors; (C) fatty acid synthase (FAS), (D) adiponectin (ADIPOQ), (E) peroxisome proliferator-activated receptor-gamma $(\mathrm{PPAR}-\gamma)$, and $(\mathrm{F})$ CCAAT-enhancer-binding protein- $\alpha(\mathrm{CEBP} / \alpha)$ in 3T3-L1 cells are shown. MDI = methylisobutylxanthine-dexamethasoneinsulin. Differences between the means compared with the positive control group were evaluated using ANOVA; ${ }^{*} P<0.05$.

group $(P<0.05$; Figure 2B). Additionally, the brown fat:BW ratio in the positive control group was not significantly different from that in the CAU 28 and simvastatin groups $(P=0.46$; Figure $2 \mathrm{C})$. However, the brown fat:white fat weight ratio was significantly increased in the negative control and CAU 28 groups relative to the positive control group (Figure 2D).

\section{Effects of Lc. chungangensis CAU 28 on TC, HDL:TC, LDL:TC, and TG Levels}

Administration of Lc. chungangensis CAU 28 and simvastatin significantly reduced TC and TG levels in HFD-fed obese mice $(P<0.05)$ after 12 wk of feeding NFD and HFD (Figure $3 \mathrm{~A}$ and $3 \mathrm{~B}$ ). The mean LDL:TC ratios in the simvastatin $(P=0.02)$ and
CAU 28 groups $(P=0.03)$ were lower than that in the positive control group, but the difference was only statistically significant for the former (Figure 3C). The mean HDL:TC ratio in the positive control group was higher than those in other experimental groups, but no statistical significance was noted among the groups $(P$ $=0.13 ;$ Figure $3 \mathrm{D})$.

\section{Effect of Lc. chungangensis CAU 28 on Inflammatory Markers in the Blood}

The mean serum leptin level was significantly higher in the positive control group than in the negative control group $(P<0.05)$, indicating that HFD led to leptin resistance (Figure 4A). The mean serum leptin level was significantly lower in the Lc. chungangensis CAU 
28 group than that in the positive control group $(P<$ $0.05)$. No statistical differences were observed between the mean serum leptin levels of the simvastatin and positive control groups $(P=0.10)$. The mean serum adiponectin level was higher in the negative control group than in the positive control group (Figure 4B). The mean serum adiponectin levels in the Lc. chungangensis CAU $28(P<0.05)$ and simvastatin $(P<0.05)$ groups were significantly higher than those in the positive control group. The mean serum levels of TNF- $\alpha$, IL- $1 \beta$, IFN- $\gamma$, and IL- 6 were significantly higher in the positive control group than in the negative control and Lc. chungangensis CAU 28 groups $(P<0.05$; Figure $4 \mathrm{C}-\mathrm{F})$. Furthermore, the mean levels of TNF- $\alpha$, IFN- $\gamma$, and IL-6 in the simvastatin group were lower than those in the positive control group $(P<0.05)$; however, IL-1 $\beta$ levels in the simvastatin and positive control groups were not significantly different $(P=0.54)$.

\section{Effect of Lc. chungangensis CAU 28 on Lymphocytes}

The mean $\mathrm{CD}^{+}{ }^{+}$T-cell count was significantly lower in the positive control group than in the negative control group $(P<0.05$; Figure 5$)$ and higher in the
CAU 28 group than in the positive control group $(P=$ 0.01 ). However, there was no significant difference between the groups in terms of $\mathrm{CD} 8^{+} \mathrm{T}$-cell count. These observations indicate that oral administration of $L c$. chungangensis $\mathrm{CAU} 28$ improved the immune response by increasing the numbers of the $\mathrm{CD} 4^{+}$helper $\mathrm{T}$ cells.

\section{Effect of Lc. chungangensis CAU 28 Intake on Hepatic Steatosis and Adipocyte Size}

Histological analysis revealed abnormal lipid accumulation in the livers of mice in the positive control group (Figure 6). In the positive control group, vesicular damage was observed in the liver tissue, and the hepatic fat content was high, with a large number of fat vacuoles close to the blood vessels, leakage of cell contents, cell swelling, and cytomembrane damage. Hepatic steatosis was less pronounced after oral administration of $L c$. chungangensis CAU 28 to HFD-induced obese mice than in the positive control group: the hepatocytes contained fewer fat vacuoles and the morphology was similar to that of the liver of mice in the negative control group. The mean brown adipocyte size was smaller in the positive control group than in the negative control group. However, no significant differences in size were
(A)

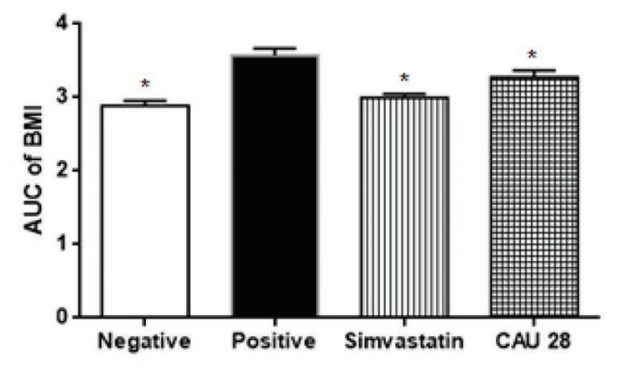

(C)

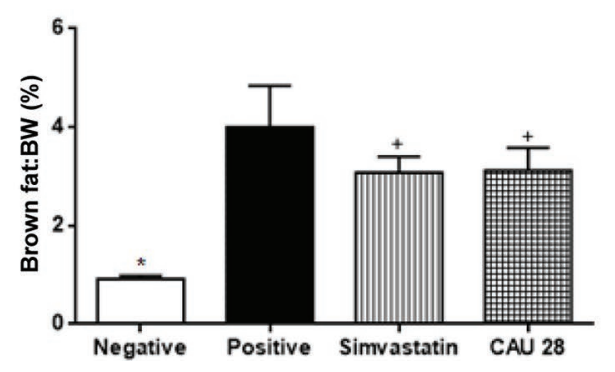

(B)

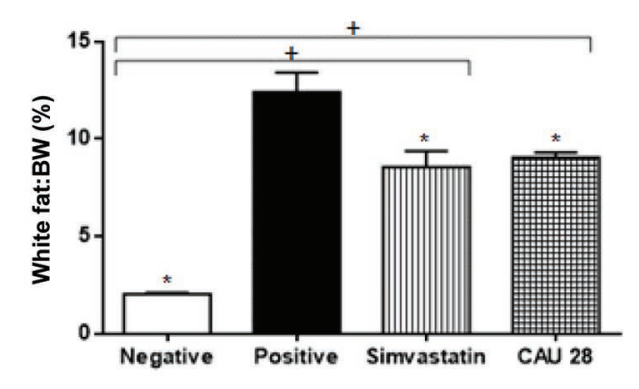

(D)

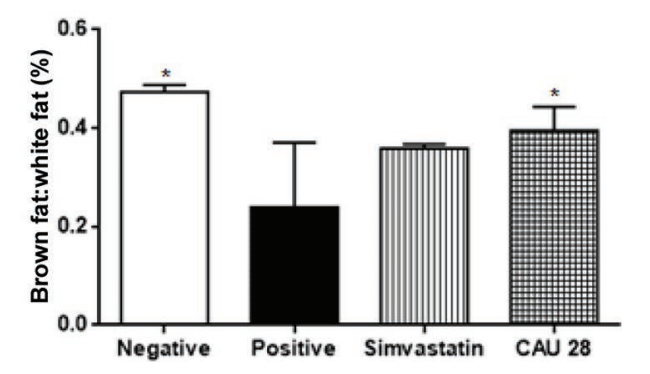

Figure 2. Effect of oral administration of Lactococcus chungangensis CAU 28 dry cells on body mass index (BMI) and adipose tissue weight of mice in obesity model: (A) BMI, (B) white fat:BW ratio, (C) brown fat:BW ratio, and (D) brown fat:white fat ratio (\%). Statistical significance was determined using ANOVA with Tukey post hoc tests; error bars indicate standard deviations. ${ }^{*} P<0.05$ vs. the positive control group, and ${ }^{+} P<0.05$ vs. the negative control group. Treatment groups: negative control group: fed normal-fat diet and orally administered PBS; positive control group: fed high-fat diet (HFD) and orally administered PBS; simvastatin group: fed HFD and orally administered simvastatin (a lipid-lowering medication that also lowers leptin levels and can effect weight loss); and CAU 28 group: fed HFD and orally administered freezedried Lactococcus chungangensis CAU 28. AUC = area under the receiver operating characteristic curve. 
(A)

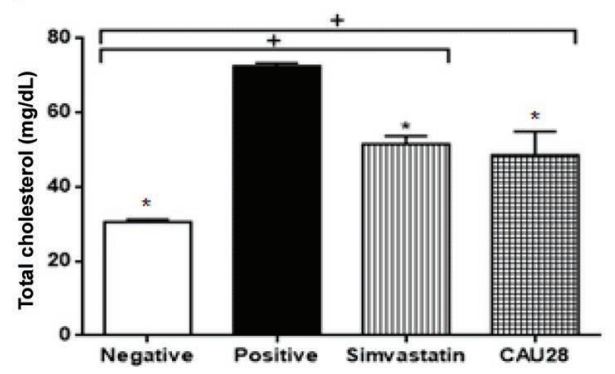

(C)

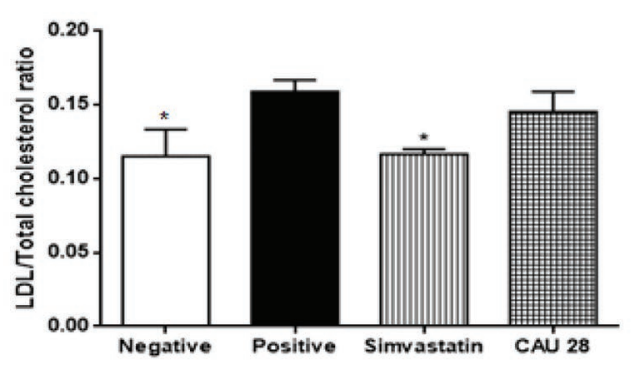

(B)

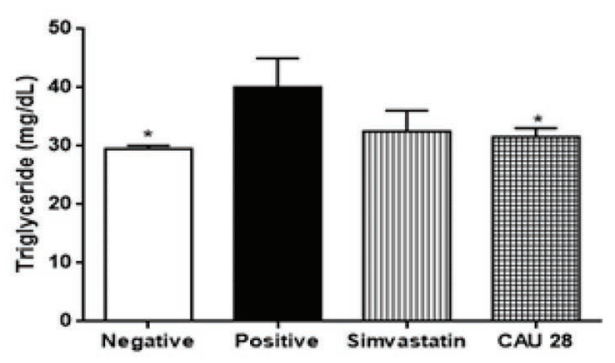

(D)

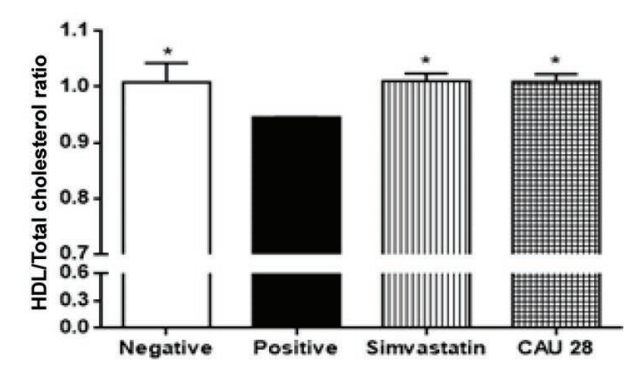

Figure 3. Effect of oral administration of Lactococcus chungangensis CAU 28 dry cells on blood lipids: (A) total cholesterol (TC), (B) triglycerides, (C) low-density lipoprotein (LDL):TC, and (D) high-density lipoprotein (HDL):TC are shown. Statistical significance was determined using ANOVA with Tukey post hoc tests; error bars indicate standard deviations. ${ }^{*} P<0.05$ vs. the positive control group, and ${ }^{+} P<0.05$ vs. the negative control group. Treatment groups: negative control group: fed normal-fat diet and orally administered PBS; positive control group: fed high-fat diet (HFD) and orally administered PBS; simvastatin group: fed HFD and orally administered simvastatin (a lipid-lowering medication that also lowers leptin levels and can effect weight loss); and CAU 28 group: fed HFD and orally administered freeze-dried Lactococcus chungangensis CAU 28.

apparent between the Lc. chungangensis CAU 28 and positive control groups. The mean abdominal adipocyte size $\left(395.7 \pm 1.9 \mu^{2}\right)$ was significantly larger in the positive control group than in all other groups $(P$ $<0.05$; negative control: $313.4 \pm 1.5 \mu \mathrm{m}^{2}$; simvastatin: $325.1 \pm 1.1 \mu \mathrm{m}^{2}$; CAU 28: $311.0 \pm 1.8 \mu \mathrm{m}^{2}$ ). Additionally, the subcutaneous adipocyte size was significantly greater in the positive control group $\left(234.8 \pm 1.7 \mu \mathrm{m}^{2}\right)$ than in the negative $\left(224.8 \pm 1.4 \mu^{2} ; P<0.05\right)$ and CAU $28\left(225.4 \pm 1.8 \mu \mathrm{m}^{2} ; P<0.05\right)$ groups. However, there was no significant difference in size between the positive control group and the simvastatin group (234.4 $\left.\pm 1.5 \mu \mathrm{m}^{2} ; P=0.38\right)$.

\section{DISCUSSION}

Recent studies have highlighted the idea that bacteria from certain genera, such as Lactobacillus and Bifidobacterium, play roles in energy metabolism and weight management in obese individuals (Kobyliak et al., 2016). Similarly, Lactococcus spp. may be used to improve human and animal health because these bacteria are generally considered nonpathogenic and safe in a food context (Holzapfel et al., 2001). As indicated in some studies, Lactococcus lactis has been linked to insulin resistance and systemic inflammation, exerting an antiobesity effect (Durack and Lynch, 2019; Sivamaruthi et al., 2019).

In the present study, we evaluated the effects of $L c$. chungangensis CAU 28 in HFD-induced obese mice and in vitro. Our data indicated that this strain could be developed into a probiotic product to reduce lipid deposition in the body and provide novel insights into the prevention and control of obesity.

The mean BMI was high among HFD-induced obese mice, whereas it was lower among mice in the $L c$. chungangensis CAU 28 group at wk 12 . It has been reported that the administration of probiotic bacteria containing some Lactobacillus strains (e.g., Lactobacillus gasseri and Lactobacillus plantarum) is associated with decreased BMI values (Sharafedtinov et al., 2013; Miyoshi et al., 2014). The white fat (abdominal and subcutaneous fat):BW ratio (8.05) was markedly lower in the Lc. chungangensis CAU 28 group than in the positive control group in our study. Previous studies have shown that Lb. gasseri BNR17 and Lactobacillus paracasei ST11 are associated with significant reductions in white fat (abdominal and subcutaneous fat):BW ratios (to ratios of 8.07. and 8.01, respectively; Tanida et al., 2008; Kang et al., 2013). These results 
are supported by previous Lactobacillus studies (Mazloom et al., 2019) and are associated with reductions in leptin levels (Harris, 2014). The presented observations indicate that, in addition to decreasing the BMI and white adipose tissue:BW ratio, Lc. chungangensis CAU 28 might prevent organ damage associated with obesity.

Lactococcus chungangensis CAU 28 intake was associated with an increased HDL:TC ratio (1.00) and decreased levels of TC (decrease of 33\%) and TG (decrease of $32 \%$ ) and LDL:TC ratio (0.15). Decreased TG and TC levels have been reported in association with Lb. plantarum HAC01 supplementation in mice, with a strain-dependent effect (Park et al., 2017). Similar to our findings of decreased TC and TG levels, a previous

(A)

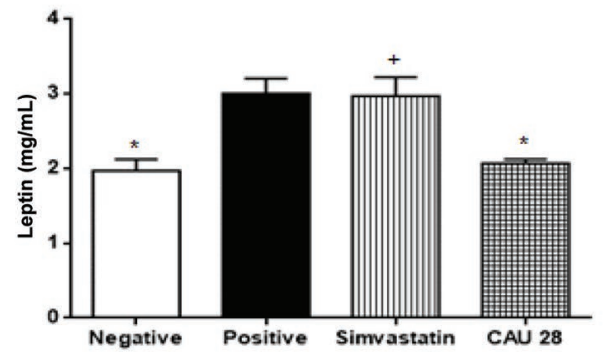

(C)

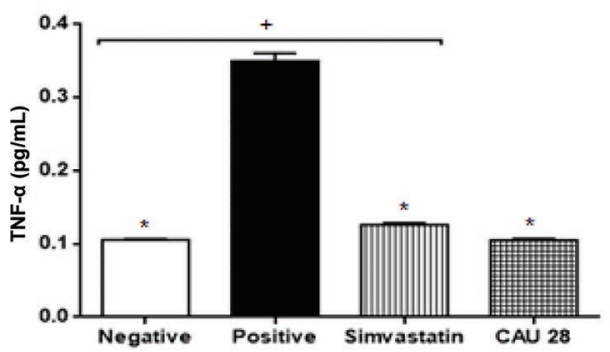

(E)

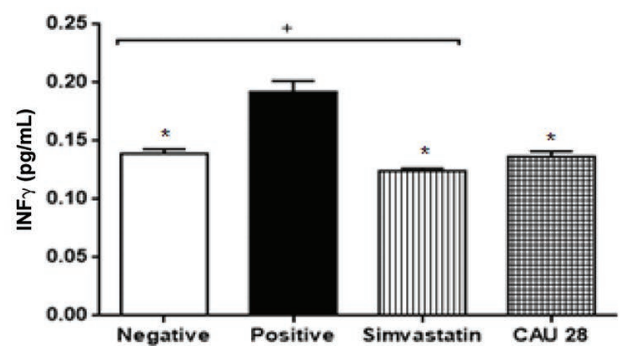

study observed decreased levels of TC (decrease of 33\%) and TG (decrease of 32\%; Jeun et al., 2010). Moreover, our results agreed with those of Jeun et al. (2010), who found that Lb. plantarum KCTC3928 was associated with the hypocholesterolemic effects of increasing the ratio of HDL:TC (0.97) and decreasing levels of TC (decrease of $32 \%$ ) and LDL:TC ratio (0.19). These results illustrate an association between lesser BW gain and reductions in mean adipocyte size, leptin levels, and cholesterol levels (Kang and Cai, 2018). Our findings indicate that intake of Lc. chungangensis CAU 28 might affect lipid metabolism.

Histopathological analysis was performed to delineate the histopathological changes associated with the different diets investigated. In NFD-fed mice, the hepa-

(B)

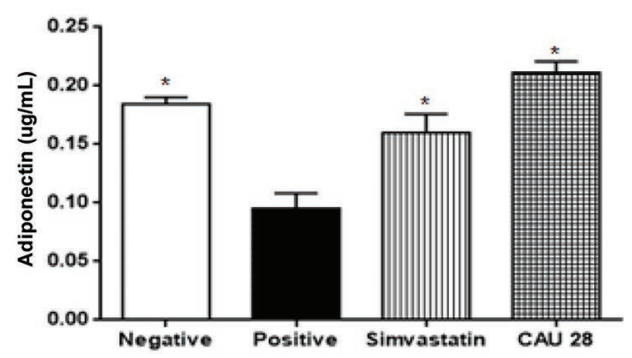

(D)

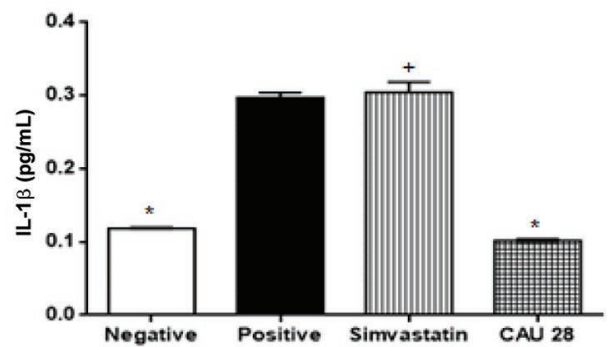

(F)

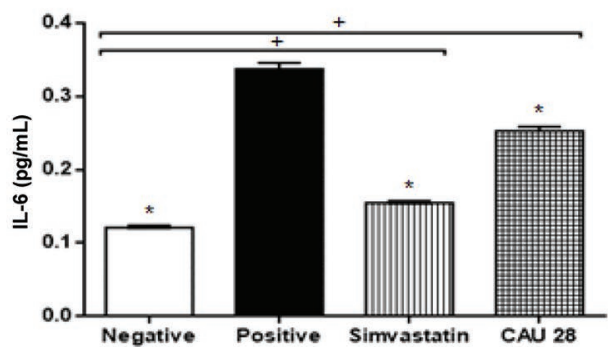

Figure 4. Effect of oral administration of Lactococcus chungangensis CAU 28 dry cells on adipokine and cytokine levels in the serum. The serum levels of the following molecules were determined using ELISA: (A) leptin, (B) adiponectin, (C) tumor necrosis factor (TNF)- $\alpha$, (D) IL-1 $\beta$, (E) IFN- $\gamma$, and (F) IL-6. Statistical significance was determined using ANOVA with Tukey post hoc tests; error bars indicate standard deviations. ${ }^{*} P<0.05$ vs. the positive control group, and ${ }^{+} P<0.05$ vs. the negative control group. Treatment groups: negative control group: fed normal-fat diet and orally administered PBS; positive control group: fed high-fat diet (HFD) and orally administered PBS; simvastatin group: fed HFD and orally administered simvastatin (a lipid-lowering medication that also lowers leptin levels and can effect weight loss); and CAU 28 group: fed HFD and orally administered freeze-dried Lactococcus chungangensis CAU 28. 


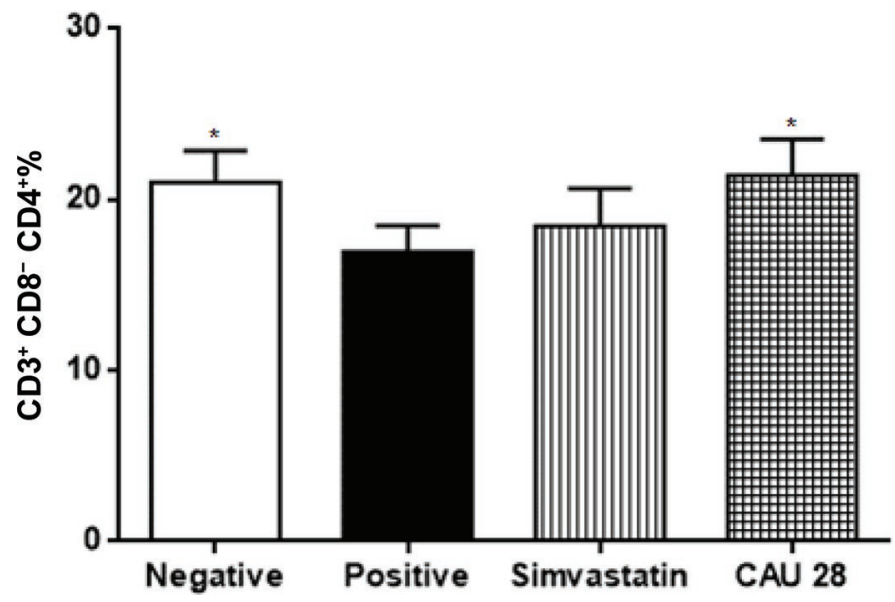

Figure 5. Effect of oral administration of Lactococcus chungangensis CAU 28 dry cells on T-cell activation evaluated using flow cytometry. The populations (\%) of helper $\mathrm{T}$ cells $\left(\mathrm{CD} 3^{+} \mathrm{CD} 8^{-} \mathrm{CD} 4^{+}\right)$ are shown. Statistical significance was determined using ANOVA with Tukey post hoc tests; error bars indicate standard deviations. ${ }^{*} P<$ 0.05 vs. positive control group. Treatment groups: negative control group: fed normal-fat diet and orally administered PBS; positive control group: fed high-fat diet (HFD) and orally administered PBS; simvastatin group: fed HFD and orally administered simvastatin (a lipid-lowering medication that also lowers leptin levels and can effect weight loss); and CAU 28 group: fed HFD and orally administered freeze-dried Lactococcus chungangensis CAU 28.

tocyte and adipocyte structures were readily observed and found to be undamaged. In the Lc. chungangensis CAU 28 group, the degree of histopathological change was similar to that in the negative control group (NFDfed mice) with respect to hepatocyte and adipocyte appearance. Liver steatosis and obesity induced by a high-fat diet are predisposing factors to oxidative stress, activating inflammatory pathways in hepatocytes (Cheng et al., 2019; Wang et al., 2020). Additionally, a reduction in fat micelles in enterocytes might lead to reduced adipocyte hypertrophy and liver steatosis, which could be associated with probiotic intake (Cano et al., 2013). Together, our results indicated that $L c$. chungangensis CAU 28 may suppress hepatocyte and adipocyte damage. Further, adipocyte damage is associated with suppression of obesity-regulated inflammatory cytokines, such as IL-6, TNF- $\alpha$, and IL-1 $\beta$ (Coppack, 2001). Previous studies have revealed reductions in proinflammatory cytokine levels and the prevention of BW gain, fat accumulation, and proinflammatory gene expression in the adipose tissue of HFD-fed mice after probiotic supplementation (Miyoshi et al., 2014; Moya-Pérez et al., 2015). In the present study, high numbers of $\mathrm{CD}^{+} \mathrm{T}$ cells were noted in the Lc. chungangensis CAU 28 group. High numbers of regulatory $\mathrm{T}$ cells $\left(\mathrm{CD} 4^{+} \mathrm{T}\right.$ cells $)$ suppress the obesity-associated systemic inflammation response and reduce proinflam- matory cytokines in adipose tissue in obese individuals (Feuerer et al., 2009; Nishimura et al., 2009; DeFuria et al., 2013; Moya-Pérez et al., 2015). Therefore, our observations might indicate that Lc. chungangensis CAU 28 effectively improved the immune response by increasing the number of $\mathrm{CD} 4^{+} \mathrm{T}$ cells and suppressing adipocyte damage and weight gain.

Serum leptin levels were reduced in the Lc. chungangensis CAU 28 group, whereas serum adiponectin levels were elevated compared with the positive control group. Adiponectin reduces the activity of $T N F-\alpha$ and inhibits IL-6 production, whereas leptin deficiency is associated with TNF- $\alpha$ activity (Forny-Germano et al., 2019). Our results were in line with previous reports attributing reduced levels of leptin and improved adiponectin secretion in obesity to probiotic supplementation (Marques et al., 1998; Boden et al., 2005; Qiao et al., 2015). Additionally, white fat adipocytes in the CAU 28 group were smaller than those in the positive control group. This could explain how Lc. chungangensis CAU 28 intake could lead to high adiponectin levels and low leptin levels, which also leads to a reduction in adipocyte size. Adipocyte size is a key factor in the expression of leptin and adiponectin. These 2 hormones are associated with systemic energy homeostasis (Stern et al., 2016). Low leptin levels lead to decreased lipid accumulation (e.g., white fat adipocytes) and increased TG hydrolysis (Kowalska et al., 2019). Further, high adiponectin maintains healthy adipose tissue and prevents lipid accumulation (Zhou et al., 2019). Our in vitro experiment showed that Lc. chungangensis CAU 28 is sufficient for preventing lipid accumulation, inhibiting TG formation, and decreasing adipogenic transcription factors (FAS, adiponectin, PPAR- $\gamma$, and C/ $\mathrm{EBP} \alpha$ ) in differentiated 3T3-L1 cells. The activation of PPAR $-\gamma$ and CEBP $/ \alpha$ leads to adipose differentiation and expression of FAS, leptin, and adiponectin (Park et al., 2014; Choi et al., 2020). Therefore, the presented results indicate that Lc. chungangensis $\mathrm{CAU} 28$ might prevent lipid accumulation and obesity through its involvement in systemic energy homeostasis mediated by leptin and adiponectin expression. In particular, $L c$. chungangensis CAU 28 administration significantly decreased the levels of TC and TG compared with simvastatin administration. Furthermore, Lc. chungangensis CAU 28 treatment dramatically decreased the serum concentration of leptin compared with simvastatin treatment. The proinflammatory cytokines TNF- $\alpha$ and IL-1 $\beta$ were affected similarly to leptin. However, adiponectin and anti-inflammatory IL-6 levels were increased to a greater extent in association with CAU 28 than with simvastatin. Therefore, Lc. chungangensis CAU 28 may have applications as a supplement for treating 


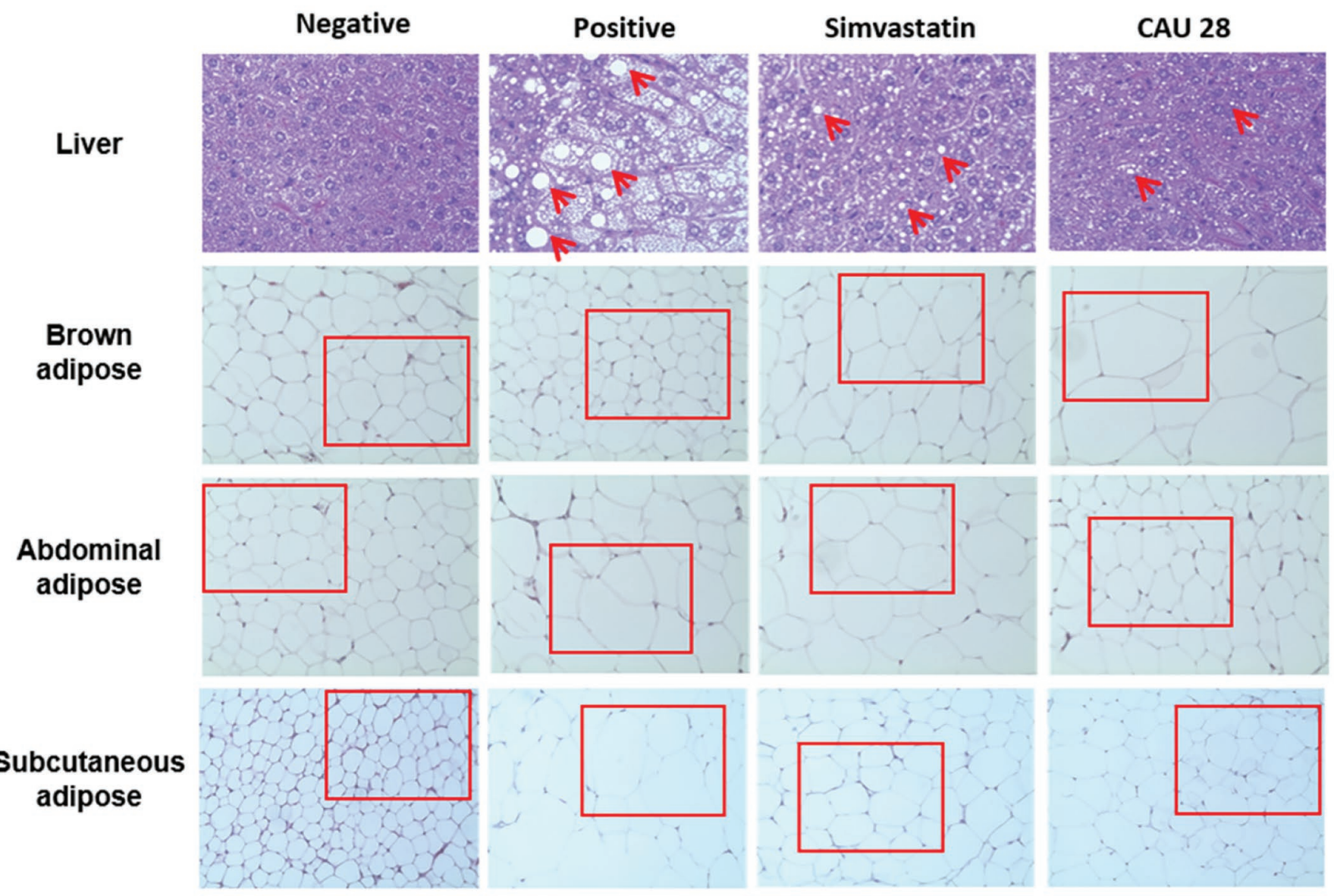

Figure 6. Effect of oral administration of Lactococcus chungangensis CAU 28 cells on liver and adipose tissue. Histological analyses of the liver, brown adipose tissue, abdominal adipose tissue, and subcutaneous adipose tissue are shown (400× magnification). Red arrows and rectangles indicate regions of interest (red arrow: liver fat vacuoles; red rectangles: adipocytes). Treatment groups: negative control group: fed normal-fat diet and orally administered PBS; positive control group: fed high-fat diet (HFD) and orally administered PBS; simvastatin group: fed HFD and orally administered simvastatin (a lipid-lowering medication that also lowers leptin levels and can effect weight loss); and CAU 28 group: fed HFD and orally administered freeze-dried Lactococcus chungangensis CAU 28.

obesity, and clinical trials to investigate its efficacy and safety are warranted.

\section{CONCLUSIONS}

This study was conducted to determine the effects of Lc. chungangensis CAU 28 on obesity in HFD-fed mice through in vitro and in vivo experiments. At the in vitro level, Lc. chungangensis CAU 28 treatment of differentiated 3T3-L1 cells prevented lipid accumulation and TG formation. Additionally, HFD-fed C57BL/6J mice were orally administered $L c$. chungangensis CAU 28 , which suppressed weight gain; increased adiponectin levels; decreased leptin, TC, and TG levels; effectively mitigated liver damage; and reduced the secretion of proinflammatory cytokines. These results suggest that Lc. chungangensis CAU 28 can be developed into a potential supplement to mitigate obesity.

\section{ACKNOWLEDGMENTS}

The authors thank YoHan Nam and Kiyoung Kim for their contributions to animal experiments. The authors confirm that they have no conflicts of interest.

\section{REFERENCES}

Blessing, M. M., T. C. Cornish, J. R. Lehman, S. M. Jenkins, J. J. Maleszewski, M. C. Aubry, and P. T. Lin. 2016. Quantitation of Oil Red $\mathrm{O}$ staining via digital imaging analysis in the evaluation for pulmonary fat embolization at autopsy. Lab. Invest. 96:4A-5A.

Boden, G., P. She, M. Mozzoli, P. Cheung, K. Gumireddy, P. Reddy, X. Xiang, Z. Luo, and N. Ruderman. 2005. Free fatty acids produce insulin resistance and activate the proinflammatory nuclear factor- $\mathrm{B}$ pathway in rat liver. Diabetes 54:3458-3465. https://doi .org/10.2337/diabetes.54.12.3458.

Cano, P. G., A. Santacruz, F. M. Trejo, and Y. Sanz. 2013. Bifidobacterium CECT 7765 improves metabolic and immunological alterations associated with obesity in high-fat diet-fed mice. Obesity (Silver Spring) 21:2310-2321. https://doi.org/10.1002/oby.20330. 
Castaner, O., A. Goday, Y. M. Park, S. H. Lee, F. Magkos, S. A. T. E. Shiow, and H. Schröder. 2018. The gut microbiome profile in obesity: A systematic review. Int. J. Endocrinol. 2018:4095789. https://doi.org/10.1155/2018/4095789.

Cheng, K., Z. Song, H. Zhang, S. Li, C. Wang, L. Zhang, and T. Wang. 2019. The therapeutic effects of resveratrol on hepatic steatosis in high-fat diet-induced obese mice by improving oxidative stress, inflammation and lipid-related gene transcriptional expression. Med. Mol. Morphol. 52:187-197. https://doi.org/10.1007/s00795 -019-00216-7.

Cho, S. L., S. W. Nam, J. H. Yoon, J. S. Lee, A. Sukhoom, and W. Kim. 2008. Lactococcus chungangensis sp. nov., a lactic acid bacterium isolated from activated sludge foam. Int. J. Syst. Evol. Microbiol. 58:1844-1849. https://doi.org/10.1099/ijs.0.65527-0.

Choi, W. J., H. J. Dong, H. U. Jeong, D. W. Ryu, S. M. Song, Y. R. Kim, H. H. Jung, T. H. Kim, and Y. H. Kim. 2020. Lactobacillus plantarum LMT1-48 exerts anti-obesity effect in high-fat diet-induced obese mice by regulating expression of lipogenic genes. Sci. Rep. 10:869. https://doi.org/10.1038/s41598-020-57615-5.

Choi, W. J., M. Konkit, Y. Kim, M. K. Kim, and W. Kim. 2016. Oral administration of Lactococcus chungangensis inhibits 2,4-dinitrochlorobenzene-induced atopic-like dermatitis in NC/Nga mice. J. Dairy Sci. 99:6889-6901. https://doi.org/10.3168/jds.2016-11301.

Coppack, S. W. 2001. Pro-inflammatory cytokines and adipose tissue. Proc. Nutr. Soc. 60:349-356. https://doi.org/10.1079/ PNS2001110.

DeFuria, J., A. C. Belkina, M. Jagannathan-Bogdan, J. Snyder-Cappione, J. D. Carr, Y. R. Nersesova, D. Markham, K. J. Strissel, A. A. Watkins, M. Zhu, J. Allen, J. Bouchard, G. Toraldo, R. Jasuja, M. S. Obin, M. E. McDonnell, C. Apovian, G. V. Denis, and B. S. Nikolajczyk. 2013. B cells promote inflammation in obesity and type 2 diabetes through regulation of T-cell function and an inflammatory cytokine profile. Proc. Natl. Acad. Sci. USA 110:5133-5138. https://doi.org/10.1073/pnas.1215840110.

Durack, J., and S. V. Lynch. 2019. The gut microbiome: Relationships with disease and opportunities for therapy. J. Exp. Med. 216:20-40. https://doi.org/10.1084/jem.20180448.

FAO/WHO. 2002. Guidelines for the Evaluation of Probiotics in Food. Working Group Report 2002. Food and Agriculture Organization of the United Nations, Rome, Italy; World Health Organization, Geneva, Switzerland.

Fasshauer, M., S. Kralisch, M. Klier, U. Lossner, M. Bluher, J. Klein, and R. Paschke. 2003. Adiponectin gene expression and secretion is inhibited by interleukin-6 in 3T3-L1 adipocytes. Biochem. Biophys. Res. Commun. 301:1045-1050. https://doi.org/10.1016/ S0006-291X(03)00090-1.

Feuerer, M., J. A. Hill, D. Mathis, and C. Benoist. 2009. Foxp3+ regulatory T cells: Differentiation, specification, subphenotypes. Nat. Immunol. 10:689-695. https://doi.org/10.1038/ni.1760.

Forny-Germano, L., F. G. De Felice, and M. N. D. N. Vieira. 2019. The role of leptin and adiponectin in obesity-associated cognitive decline and Alzheimer's disease. Front. Neurosci. 12:1027. https:// doi.org/10.3389/fnins.2018.01027.

Golubnitschaja, O., and V. Costigliola. 2012. General report \& recommendations in predictive, preventive and personalised medicine 2012: White paper of the European Association for Predictive, Preventive and Personalised Medicine. EPMA J. 3:14. https://doi .org/10.1186/1878-5085-3-14.

Gwon, S. Y., J. Y. Ahn, C. H. Jung, B. K. Moon, and T. Y. Ha. 2013. Shikonin suppresses ERK $1 / 2$ phosphorylation during the early stages of adipocyte differentiation in 3T3-L1 cells. BMC Complement. Altern. Med. 13:207. https://doi.org/10.1186/1472-6882-13 $-207$.

Harris, R. B. 2014. Direct and indirect effects of leptin on adipocyte metabolism. Biochim. Biophys. Acta 1842:414-423. https://doi .org/10.1016/j.bbadis.2013.05.009.

Holzapfel, W. H., P. Haberer, R. Geisen, J. Björkroth, and U. Schillinger. 2001. Taxonomy and important features of probiotic microorganisms in food and nutrition. Am. J. Clin. Nutr. 73:365S-373S. https://doi.org/10.1093/ajcn/73.2.365s.
Horowitz, J. F., S. W. Coppack, D. Paramore, P. E. Cryer, G. Zhao, and S. Klein. 1999. Effect of short-term fasting on lipid kinetics in lean and obese women. Am. J. Physiol. 276:E278-E284. https:// doi.org/10.1152/ajpendo.1999.276.2.E278.

Hursting, S. D., J. DiGiovanni, A. J. Dannenberg, M. Azrad, D. LeRoith, W. Demark-Wahnefried, M. Kakarala, A. Brodie, and N. A. Berger. 2012. Obesity, energy balance, and cancer: New opportunities for prevention. Cancer Prev. Res. (Phila.) 5:1260-1272. https:/ /doi.org/10.1158/1940-6207.CAPR-12-0140.

Interator, H., Y. Lebenthal, M. Hoshen, I. Safra, R. Balicer, M. Leshno, and R. Shamir. 2017. Distinct lipoprotein curves in normal weight, overweight, and obese children and adolescents. J. Pediatr. Gastroenterol. Nutr. 65:673-680. https://doi.org/10.1097/MPG .0000000000001674 .

Jeong, H. J., S. W. Park, H. Kim, S. Park, and D. Yoon. 2010. Coculture with BJ fibroblast cells inhibits the adipogenesis and lipogenesis in 3T3-L1 cells. Biochem. Biophys. Res. Commun. 392:520525. https://doi.org/10.1016/j.bbrc.2009.12.184.

Jeun, J., S. Kim, S. Y. Cho, H. J. Jun, H. J. Park, J. G. Seo, M. J. Chung, and S. J. Lee. 2010. Hypocholesterolemic effects of Lactobacillus plantarum KCTC3928 by increased bile acid excretion in C57BL/6 mice. Nutrition 26:321-330. https://doi.org/10.1016/ j.nut.2009.04.011.

Jin, D., Y. Xu, X. Mei, Q. Meng, Y. Gao, B. Li, and Y. Tu. 2013. Antiobesity and lipid lowering effects of theaflavins on high-fat diet induced obese rats. J. Funct. Foods 5:1142-1150. https://doi.org/ 10.1016/j.jff.2013.03.011.

Kang, J. H., S. I. Yun, M. H. Park, J. H. Park, S. Y. Jeong, and H. O. Park. 2013. Anti-obesity effect of Lactobacillus gasseri BNR17 in high-sucrose diet-induced obese mice. PLoS One 8:e54617. https:/ /doi.org/10.1371/journal.pone.0054617.

Kang, Y., and Y. Cai. 2018. The development of probiotics therapy to obesity: A therapy that has gained considerable momentum. Hormones (Athens) 17:141-151. https://doi.org/10.1007/s42000 -018-0003-y.

Kim, J. H., K. Kim, and W. Kim. 2019. Cream cheese-derived Lactococcus chungangensis CAU 28 modulates the gut microbiota and alleviates atopic dermatitis in BALB/c mice. Sci. Rep. 9:446. https://doi.org/10.1038/s41598-018-36864-5.

Kobyliak, N., C. Conte, G. Cammarota, A. P. Haley, I. Styriak, L. Gaspar, J. Fusek, L. Rodrigo, and P. Kruzliak. 2016. Probiotics in prevention and treatment of obesity: A critical view. Nutr. Metab. (Lond.) 13:14. https://doi.org/10.1186/s12986-016-0067-0.

Konkit, M., W. J. Choi, and W. Kim. 2015. Alcohol dehydrogenase activity in Lactococcus chungangensis: Application in cream cheese to moderate alcohol uptake. J. Dairy Sci. 98:5974-5982. https:// doi.org/10.3168/jds.2015-9697.

Konkit, M., W. J. Choi, and W. Kim. 2016. Aldehyde dehydrogenase activity in Lactococcus chungangensis: Application in cream cheese to reduce aldehyde in alcohol metabolism. J. Dairy Sci. 99:17551761. https://doi.org/10.3168/jds.2015-10549.

Konkit, M., J. H. Kim, N. Bora, and W. Kim. 2014. Transcriptomic analysis of Lactococcus chungangensis sp. nov. and its potential in cheese making. J. Dairy Sci. 97:7363-7372. https://doi.org/10 .3168/jds.2014-8299.

Konkit, M., and W. Kim. 2016. Activities of amylase, proteinase, and lipase enzymes from Lactococcus chungangensis and its application in dairy products. J. Dairy Sci. 99:4999-5007. https://doi.org/10 .3168/jds.2016-11002.

Kowalska, K., A. Olejnik, J. Zielińska-Wasielica, and M. Olkowicz. 2019. Raspberry (Rubus idaeus L.) fruit extract decreases oxidation markers, improves lipid metabolism and reduces adipose tissue inflammation in hypertrophied 3T3-L1 adipocytes. J. Funct. Foods 62:103568. https://doi.org/10.1016/j.jff.2019.103568.

Lee, H. S., H. J. Lee, and H. J. Suh. 2011. Silk protein hydrolysate increases glucose uptake through up-regulation of GLUT 4 and reduces the expression of leptin in 3T3-L1 fibroblast. Nutr. Res. 31:937-943. https://doi.org/10.1016/j.nutres.2011.09.009.

Marques, B. G., D. B. Hausman, and R. J. Martin. 1998. Association of fat cell size and paracrine growth factors in development of hy- 
perplastic obesity. Am. J. Physiol. 275:R1898-R1908. https://doi .org/10.1152/ajpregu.1998.275.6.R1898.

Mazloom, K., I. Siddiqi, and M. Covasa. 2019. Probiotics: How effective are they in the fight against obesity? Nutrients 11:258. https: //doi.org/10.3390/nu11020258

Miyoshi, M., A. Ogawa, S. Higurashi, and Y. Kadooka. 2014. Antiobesity effect of Lactobacillus gasseri SBT2055 accompanied by inhibition of pro-inflammatory gene expression in the visceral adipose tissue in diet-induced obese mice. Eur. J. Nutr. 53:599-606. https://doi.org/10.1007/s00394-013-0568-9.

Moya-Pérez, A., A. Neef, and Y. Sanz. 2015. Bifidobacterium pseudocatenulatum CECT 7765 reduces obesity-associated inflammation by restoring the lymphocyte-macrophage balance and gut microbiota structure in high-fat diet-fed mice. PLoS One 10:e0126976. https:/ /doi.org/10.1371/journal.pone.0126976.

Naudin, C. R., K. Maner-Smith, J. A. Owens, G. M. Wynn, B. S. Robinson, J. D. Matthews, A. R. Reedy, L. Luo, A. Wolfarth, T. M. Darby, E. A. Ortlund, and R. M. Jones. 2020. Lactococcus lactis ssp. cremoris elicits protection against metabolic changes induced by a western-style diet. Gastroenterology. https://doi.org/ 10.1053/j.gastro.2020.03.010. In press.

Nguyen, T. L., W. K. Chun, A. Kim, N. Kim, H. J. Roh, Y. Lee, M. Yi, S. Kim, C. I. Park, and D. H. Kim. 2018. Dietary probiotic effect of Lactococcus lactis WFLU12 on low-molecular-weight metabolites and growth of olive flounder (Paralichythys olivaceus). Front. Microbiol. 9:2059. https://doi.org/10.3389/fmicb.2018.02059.

Nishimura, S., I. Manabe, M. Nagasaki, K. Eto, H. Yamashita, M. Ohsugi, M. Otsu, K. Hara, K. Ueki, S. Sugiura, K. Yoshimura, T. Kadowaki, and R. Nagai. 2009. CD8+ effector T cells contribute to macrophage recruitment and adipose tissue inflammation in obesity. Nat. Med. 15:914-920. https://doi.org/10.1038/nm.1964.

Park, J. E., S. H. Oh, and Y. S. Cha. 2014. Lactobacillus plantarum LG 42 isolated from gajami sik-hae decreases body and fat pad weights in diet-induced obese mice. J. Appl. Microbiol. 116:145156. https://doi.org/10.1111/jam.12354.

Park, S., Y. Ji, H. Y. Jung, H. Park, J. Kang, S. H. Choi, H. Shin, C. K. Hyun, K. T. Kim, and W. H. Holzapfel. 2017. Lactobacillus plantarum HAC01 regulates gut microbiota and adipose tissue accumulation in a diet-induced obesity murine model. Appl. Microbiol. Biotechnol. 101:1605-1614. https://doi.org/10.1007/s00253 -016-7953-2.

Qiao, Y., J. Sun, S. Xia, L. Li, Y. Li, P. Wang, Y. Shi, and G. Le. 2015. Effects of different Lactobacillus reuteri on inflammatory and fat storage in high-fat diet-induced obesity mice model. J. Funct. Foods 14:424-434. https://doi.org/10.1016/j.jff.2015.02.013.

Rinaldi, A. E. M., E. P. De Oliveira, F. Moreto, G. F. C. P. Gabriel, J. E. Corrente, and R. C. Burini. 2012. Dietary intake and blood lipid profile in overweight and obese schoolchildren. BMC Res. Notes 5:598. https://doi.org/10.1186/1756-0500-5-598.

Rudnicki, M., G. Abdifarkosh, O. Rezvan, E. Nwadozi, E. Roudier, and T. L. Haas. 2018. Female mice have higher angiogenesis in perigonadal adipose tissue than males in response to high-fat diet. Front. Physiol. 9:1452. https://doi.org/10.3389/fphys.2018.01452.

Sharafedtinov, K. K., O. A. Plotnikova, R. I. Alexeeva, T. B. Sentsova, E. Songisepp, J. Stsepetova, I. Smidt, and M. Mikelsaar. 2013. Hypocaloric diet supplemented with probiotic cheese improves body mass index and blood pressure indices of obese hyperten- sive patients - A randomized double-blind placebo-controlled pilot study. Nutr. J. 12:138. https://doi.org/10.1186/1475-2891-12-138.

Sivamaruthi, B. S., P. Kesika, N. Suganthy, and C. Chaiyasut. 2019. A review on role of microbiome in obesity and antiobesity properties of probiotic supplements. BioMed Res. Int. 2019:3291367. https:// doi.org/10.1155/2019/3291367.

Spor, A., O. Koren, and R. Ley. 2011. Unravelling the effects of the environment and host genotype on the gut microbiome. Nat. Rev. Microbiol. 9:279-290. https://doi.org/10.1038/nrmicro2540.

Stern, J. H., J. M. Rutkowski, and P. E. Scherer. 2016. Adiponectin, leptin, and fatty acids in the maintenance of metabolic homeostasis through adipose tissue crosstalk. Cell Metab. 23:770-784. https: //doi.org/10.1016/j.cmet.2016.04.011.

Tanida, M., J. Shen, K. Maeda, Y. Horii, T. Yamano, Y. Fukushima, and K. Nagai. 2008. High-fat diet-induced obesity is attenuated by probiotic strain Lactobacillus paracasei ST11 (NCC2461) in rats. Obes. Res. Clin. Pract. 2:159-169. https://doi.org/10.1016/j.orcp 2008.04.003.

Tran, B. T., B. Y. Jeong, and J. K. Oh. 2017. The prevalence trend of metabolic syndrome and its components and risk factors in Korean adults: Results from the Korean National Health and Nutrition Examination Survey 2008-2013. BMC Public Health 17:71. https: //doi.org/10.1186/s12889-016-3936-6.

Wang, P., X. Gao, Y. Li, S. Wang, J. Yu, and Y. Wei. 2020. Bacillus natto regulates gut microbiota and adipose tissue accumulation in a high-fat diet mouse model of obesity. J. Funct. Foods 68:103923. https://doi.org/10.1016/j.jff.2020.103923.

World Health Organization. 2000. Obesity: Preventing and managing the global epidemic. WHO Technical Report Series 894. World Health Organization, Geneva, Switzerland.

World Health Organization. 2018. Obesity Overweight. World Health Organization, Geneva, Switzerland.

You, J. S., Y. J. Lee, K. S. Kim, S. H. Kim, and K. J. Chang. 2014. Ethanol extract of lotus (Nelumbo nucifera) root exhibits an antiadipogenic effect in human pre-adipocytes and anti-obesity and anti-oxidant effects in rats fed a high-fat diet. Nutr. Res. 34:258267. https://doi.org/10.1016/j.nutres.2014.01.003.

Yu, Y., T. South, and X. F. Huang. 2009. Inter-meal interval is increased in mice fed a high whey, as opposed to soy and gluten, protein diet. Appetite 52:372-379. https://doi.org/10.1016/j.appet 2008.11.011.

Zhao, B., Y. Cui, X. Fan, P. Qi, C. Liu, X. Zhou, and X. Zhang. 2019 Anti-obesity effects of Spirulina platensis protein hydrolysate by modulating brain-liver axis in high-fat diet fed mice. PLoS One 14:e0218543. https://doi.org/10.1371/journal.pone.0218543.

Zhou, Q., H. Xiang, A. Li, W. Lin, Z. Huang, J. Guo, P. Wang, Y. Chi, K. Xiang, Y. Xu, L. Zhou, K. So, X. Chen, X. Sun, and Y. Ren. 2019. Activating adiponectin signaling with exogenous AdipoRon reduces myelin lipid accumulation and suppresses macrophage recruitment after spinal cord injury. J. Neurotrauma 36:903-918. https://doi.org/10.1089/neu.2018.5783.

\section{ORCIDS}

Wonyong Kim @ https://orcid.org/0000-0001-9649-3919 